Check for updates

Cite this: RSC Adv., 2017, 7, 50643

Received 1st October 2017

Accepted 23rd October 2017

DOI: 10.1039/c7ra10859a

rsc.li/rsc-advances

\section{Thermo-activatable PNIPAM-functionalized lanthanide-doped upconversion luminescence nanocomposites used for in vitro imaging $\dagger$}

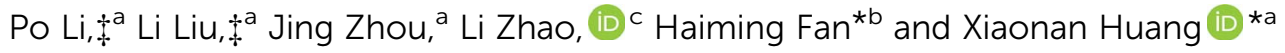

Stimuli-responsive nanocomposite has a great potential application for cell imaging, drug delivery, and improving therapeutic effect. Rare-earth upconversion nanoparticles (UCNPs) have exhibited an excellent properties as a promising biological luminescent labels for fluorescence imaging. In this work, we prepared $\beta-\mathrm{NaLuF}_{4}: \mathrm{Yb}^{3+}, \mathrm{Er}^{3+}$ UCNPs coated with thermo-responsive copolymer poly( $\mathrm{N}$-isopropyl acrylamide-Coacrylic acid) (P(NIPAM-CO-AA)) by using ligand exchange strategy to obtain novel nanocomposite UCNP@P(NIPAM-CO-AA). The acrylic acid moieties of P(NIPAM-CO-AA) coordinate with the surfaces of UCNPs, and the PNIPAM moieties can induce the UCNPs dispersion or aggregation in the aqueous solution below and above the lower critical solution temperature (LCST) of PNIPAM. The UCNPAP(NIPAM-CO-AA) exhibited weak upconversion luminescence(UCL) fluorescence intensity below the LCST but an 80 -fold enhanced $\mathrm{UCL}$ intensity at $31^{\circ} \mathrm{C}$ above their LCST with only $1{ }^{\circ} \mathrm{C}$ temperature transition range. The novel nanocomposite also shows a remarkable reversible property in aqueous solution and low cytotoxicity. For in vitro imaging, a 13-fold signal-to-noise ratio can be obtained below and above the LCST for the novel thermo-activatable UCL imaging, which implies potential application for intelligent biomedical agent.

\section{Introduction}

Recently, fluorescence imaging has become an essential tool for tumor diagnosis, biosensor and molecular detection. Lanthanide-doped upconversion nanoparticles (UCNPs), which can be excited with low-power continuous wave near-infrared (NIR) sources and emit upconversion luminescence (UCL) fluorescence at shorter wavelengths, have been paid great attention in the fields of biology and biomedicine, such as therapeutics, ${ }^{\mathbf{1 - 3}}$ molecular detection, ${ }^{\mathbf{4 - 8}}$ biological imaging ${ }^{9-11}$ and biolabeling. ${ }^{12}$ UCNPs with intelligent properties including low autofluorescence background, deep tissue penetration, reduced photodamage and higher signal-to-noise ratio (SNR) are desirable to be used for biomedical imaging. ${ }^{\mathbf{1 3}, \mathbf{1 4}}$ Several efforts have been made on enhancing the upconversion emission of UCNPs, such as increasing the interaction between upconverting lanthanide pairs $\left(e . g\right.$., $\left.\mathrm{Yb}^{3+} / \mathrm{Er}^{3+}\right)$ with the surface

${ }^{a}$ Department of Chemistry, Capital Normal University, 105 West 3rd Ring North Rd, Beijing 100048, PR China. E-mail: huangxn@cnu.edu.cn

${ }^{b}$ School of Petroleum Engineering, China University of Petroleum (East China), Qingdao 266580, Shandong Province, PR China. E-mail: haimingfan@126.com

'School of Food and Chemical Engineering, Beijing Technology and Business University, PR China

$\dagger$ Electronic supplementary information (ESI) available: TEM images, XRD spectrum, EDX analysis and up-conversion luminescence spectra of UCNP-OA. FTIR and TGA of UCNP@OA, P(NIPAM-co-AA), UCNP@ P(NIPAM-co-AA). See DOI: $10.1039 / \mathrm{c} 7 \mathrm{ra} 10859 \mathrm{a}$

$\$$ These authors contributed equally to this work. plasmon of noble metals. However, through these methods, the enhancement of the upconversion emission can only be limited in 2-12 fold..$^{15}$

Fabrication of complex nanocomposites with combination of properties is of great interests for biomedical and bioengineering applications. The stimuli-responsive polymers are often used to functionalize the nanoparticles to control their assembly, and consequently combine the properties of the individual components to illustrate novel properties. ${ }^{16-18}$ Thermoresponsive polymers with lower critical solution temperature (LCST) have been extensively investigated in both scientific and applied research areas. ${ }^{\mathbf{1 9 2 0}}$ Poly( $N$-isopropyl acrylamide) (PNIPAM) have phase transitions to undergo a drastic reversible collapse in water above their LCST, ${ }^{\mathbf{2 1 , 2 2}}$ thus was used to synthesize many multifunctional intelligent materials which can response to tiny temperature change.

In this work, we prepared a novel thermo-activatable $\beta$ $\mathrm{NaLuF}_{4}: \mathrm{Yb}^{3+}, \mathrm{Er}^{3+}$ UCNPs coated with thermo-responsive copolymer poly( $\mathrm{N}$-isopropyl acrylamide-co-acrylic acid) (P(NIPAM-co-AA)) by using ligand exchange strategy. The acrylic acid moieties of P(NIPAM-co-AA) can be coordinated with UCNPs surfaces, and the upconversion luminescence property of obtained nanocomposite UCNP@P(NIPAM-co-AA) in aqueous solution was characterized by UCL spectra. The association and dissociation of PNIPAM segment can induce the phase transition of UCNPs, thus render a fast and ultrasensitive response at LCST, and consequently the UCL intensity can have a great enhancement to increase the SNR for in vitro imaging. 


\section{Experimental}

\subsection{Materials preparation}

$\mathrm{NH}_{4} \mathrm{~F}, \mathrm{NaOH}$, ethanol, cyclohexane, and hydrochloric acid solution, 2,2-azobisisobutyronitrile (AIBN) were purchased from Sinopharm Chemical Reagent Co., China. Oleic acid (OA) and octadecene (ODE), $N$-isopropyl acrylamide (NIPAM), acrylic acid (AA) were purchased from Alfa Aesar Ltd. $\mathrm{LnCl}_{3}(>99.9 \%$, $\mathrm{Ln}=\mathrm{Lu}, \mathrm{Yb}$, and Er) was purchased from Aladin Reagent Co., China. Other chemical reagents were directly used without further purification with analytical grade. Deionized water was used throughout experiments.

\subsection{Materials characterizations}

The transmission electron microscopy (TEM) images were collected on a Hitachi 7650 at an acceleration voltage of $80 \mathrm{kV}$. The crystal phases of UCNP@OA were identified with X-ray diffraction (XRD) on a Bruker D8 Advance X-ray diffractometer with a $2 \theta$ range from $10^{\circ}$ to $70^{\circ}$ at a scanning rate of $4^{\circ}$ per minute. Energy dispersive X-rays spectroscopy (EDX) was carried out by using a Hitachi S-4800. Infrared spectra were obtained using a Spectrum One FTIR spectrometer (PerkinElmer) fitted with an attenuated total reflectance (ATR) accessory under unforced conditions and the measurements were collected at $8 \mathrm{~cm}^{-1}$ resolution and 4 scans per spectrum. Thermogravimetric analysis (TGA) of UCNP@OA, P(NIPAM-co-AA) and UCNP@P(NIPAM-co-AA) were carried out with a Q50 thermogravimetric analyzer under nitrogen flow with a heating rate of $10{ }^{\circ} \mathrm{C} \mathrm{min}^{-1}$. The upconversion fluorescence spectra were measured using a Maya 2000 Pro spectrophotometer (Shanghai Oceanhood Opto-electronics tech Co. LTD) equipped with an external $980 \mathrm{~nm}$ laser instead of the internal excitation source.

\subsection{Synthesis of nanocomposite UCNP@P(NIPAM-co-AA)}

2.3.1 Synthesis of UCNP@OA. Monodispersed OA-capped upconversion luminescence $\beta-\mathrm{NaLuF}_{4}: \mathrm{Yb}^{3+}, \mathrm{Er}^{3+}$ nanoparticles (UCNP@OA) were synthesized through a solvothermal decomposition method as reported. ${ }^{23-25} \mathrm{LuCl}_{3}(0.8 \mathrm{mmol}), \mathrm{YbCl}_{3}$ $(0.18 \mathrm{mmol})$, and $\mathrm{ErCl}_{3}(0.02 \mathrm{mmol})$ were mixed with $12 \mathrm{~mL}$ of $\mathrm{OA}$ and $15 \mathrm{~mL}$ of ODE and then added into $100 \mathrm{~mL}$ flask under stirring. The solution was heated to $150{ }^{\circ} \mathrm{C}$ for $60 \mathrm{~min}$ under argon atmosphere and then cooled down to room temperature. $\mathrm{NH}_{4} \mathrm{~F}(0.148 \mathrm{~g}, 4.0 \mathrm{mmol})$ and $\mathrm{NaOH}(0.1 \mathrm{~g}, 2.5 \mathrm{mmol})$ was slowly added into the flask, degassed at $100{ }^{\circ} \mathrm{C}$ for $30 \mathrm{~min}$, then heated to $320{ }^{\circ} \mathrm{C}$ under argon and vigorously stirred for $1 \mathrm{~h}$. Finally, the solution was cooled at room temperature, and the UCNP@OA were precipitated by centrifugation $(10000 \mathrm{rpm}$, $\left.10 \mathrm{~min}, 25^{\circ} \mathrm{C}\right)$. Later on, the UCNP@OA was washed three times with a (43.5: $40.5: 16 \mathrm{v} / \mathrm{v} / \mathrm{v})$ hexane/acetone/methanol solution.

2.3.2 Synthesis of P(NIPAM-co-AA). P(NIPAM-co-AA) was synthesized by radical polymerization method. NIPAM $(0.90 \mathrm{~g}, 8 \mathrm{mmol})$, AA (0.14 g, $2 \mathrm{mmol})$ and initiator AIBN (6.4 mg, $0.1 \mathrm{mmol}$ ) were charged into a polymerization tube. Then DMF $(5 \mathrm{~mL})$ was added to dissolve the monomer and initiator. After three cycles of freeze-pump-thaw to remove oxygen, the tube was sealed in vacuo. The polymerization was carried out at $60^{\circ} \mathrm{C}$ for 24 hours. After polymerization, the reaction mixture was dialyzed in distilled water and lyophilized to obtain a white powder.

2.3.3 Complexation of nanocomposite UCNP@P(NIPAMco-AA). The UCNP@P(NIPAM-co-AA) were prepared by using a modified ligand exchange strategy. In a typical ligand exchange procedure, $50 \mathrm{mg}$ of $\mathrm{P}(\mathrm{NIPAM}-\mathrm{co}-\mathrm{AA})$ was first dissolved in $10 \mathrm{~mL}$ of water and vigorously stirred for $30 \mathrm{~min}$ at $25{ }^{\circ} \mathrm{C}$. UCNP@OA $(10 \mathrm{mg})$ was dispersed in a mixed solution of cyclohexane $(10 \mathrm{~mL})$ and $\mathrm{DMF}(5 \mathrm{~mL})$, and then, the mixture was dropwise added into the $\mathrm{P}(\mathrm{NIPAM}-\mathrm{Co}-\mathrm{AA})$ solution at $25^{\circ} \mathrm{C}$ at $\mathrm{pH} 8.0$ and stirred for $4 \mathrm{~h}$. After cooling to room temperature, the lower layer liquid was collected until the mixture was layered. UCNP@P(NIPAM-co-AA) was obtained by centrifugation at $15000 \mathrm{rpm}$ for $5 \mathrm{~min}$. Finally, the product was washed with ethanol twice and redispersed in $10 \mathrm{~mL}$ of water.

\subsection{UCL imaging in vitro}

For the upconversion luminescent imaging, the microplate was covered with a $2 \mathrm{~mm}$ thick of chicken tissue (it was purchased in the Wumart's supermarket in Beijing). Upconversion luminescence images of $1 \mathrm{mg} \mathrm{mL}^{-1} \mathrm{UCNP} @ \mathrm{P}(\mathrm{NIPAM}-\mathrm{co}$-AA) solution plates covered with chicken tissue were acquired with an in vivo imaging system at different temperature using a $980 \mathrm{~nm}$ laser as the excitation sources, and intensities of UCL signals were analyzed with the Image J Software. Upconversion luminescence signals were detected in the green channel (520-560 nm) and red channel (600-700 $\mathrm{nm})$ at same laser power density during imaging.

\subsection{Cytotoxicity assay}

Cytotoxicity of UCNP@P(NIPAM-co-AA) was determined by the MTT assay with A549 cells (it was purchased by the Institute of Materia Medica, Chinese Academy of Medical Sciences \& Peking Union Medical College). A549 cells were seeded into a 96-well cell culture plate at $5 \times 10^{4}$ per well and incubated at $37{ }^{\circ} \mathrm{C}$ under a humidified atmosphere containing $5 \% \quad \mathrm{CO}_{2}$. The cultured medium was modified with $15 \%$ FBS, 100 units per $\mathrm{mL}$ penicillin, and 100 unit streptomycin. After incubation for $24 \mathrm{~h}$, the cultured medium with UCNP@P(NIPAM-co-AA) of different concentrations of $0,50,100,200,400 \mu \mathrm{g} \mathrm{mL}{ }^{-1}$ were added to the wells. The cells were subsequently incubated for $24 \mathrm{~h}$ then $20 \mu \mathrm{L}$ of MTT ( $5 \mathrm{mg} \mathrm{mL}^{-1}$ ) solution was added to each well and the plate was incubated for an additional $4 \mathrm{~h}$. After the addition of dimethyl sulfoxide (DMSO, $200 \mu \mathrm{L}$ per well), the assay plate was allowed to stand at room temperature for $30 \mathrm{~min}$ and was measured by means with background subtraction at $540 \mathrm{~nm}$ on a Thermo Scientific multiskan FC microplate reader.

\section{Results and discussion}

\subsection{Preparation and characterization of UCNP@P(NIPAM-} co-AA)

$\beta-\mathrm{NaLuF}_{4}: \mathrm{Yb}^{3+}, \mathrm{Er}^{3+}$ nanoparticles were synthesized following a previously reported protocol with some modifications. ${ }^{23-25}$ 
Fig. 1(a) shows transmission electron microscopy (TEM) images obtained for the monodisperse nanoparticles with a uniform size of approximately $35 \mathrm{~nm}$. And the XRD pattern of the UCNP@OA (Fig. 1(b)) revealed the formation of mainly the hexagonal phase structure as bulk $\beta-\mathrm{NaLuF}_{4}$ (Joint Committee on Powder Diffraction Standards, card no.: JCPDS 16-0334). The atomic ratios of lanthanides in the nanoparticles were obtained by energy dispersive X-ray experiments (EDX) and were $\mathrm{NaLuF}_{4}$ (77\%): $\mathrm{Yb}(20 \%), \operatorname{Er}(3 \%)$ (Fig. 1(c)). UCL spectrum of $\mathrm{NaLuF}_{4}$ :- $^{-}$ $\mathrm{Yb}^{3+}, \mathrm{Er}^{3+}$ nanoparticles in organic solution was investigated at room temperature under $980 \mathrm{~nm}$ excitation. As shown in Fig. 1(d), UCNP@OA exhibited three characteristic intense emission bands centered at 520, 540, and $653 \mathrm{~nm}$ derived from the ${ }^{2} \mathrm{H}_{11 / 2} \rightarrow{ }^{4} \mathrm{I}_{15 / 2},{ }^{4} \mathrm{~S}_{3 / 2} \rightarrow{ }^{4} \mathrm{I}_{15 / 2}$, and ${ }^{4} \mathrm{~F}_{9 / 2} \rightarrow{ }^{4} \mathrm{I}_{15 / 2}$ transitions of $\mathrm{Er}^{3+}$, respectively, where the dopant $\mathrm{Yb}^{3+}$ acted as an NIR absorber and efficiently transferred energy to the emitter $\mathrm{Er}^{3+}{ }^{36}$

The copolymer P(NIPAM-co-AA) was synthesized by conventional radical polymerization with NIPAM and AA monomer and then coordinated to the surface of the UCNPs under basic environment in aqueous solution through the carboxylic group on the polymer. In order to corroborate the efficient coordination by the copolymer P(NIPAM-co-AA), FTIR spectra were used to present the IR absorption of samples before and after surface modification of the UCNPs(Fig. S1 $†$ ). For the UCNP@OA, the multipeak bands at 2925 and $2855 \mathrm{~cm}^{-1}$ can be assigned to the stretching vibrations of $\mathrm{CH}_{2}$. After the surface modification with $\mathrm{P}$ (NIPAM-co-AA), two strong peaks at 1638 and $1518 \mathrm{~cm}^{-1}$, found in the FT-IR spectrum of UCNP@P(NIPAM-co-AA), correspond to the amide bending vibration and carboxylic symmetrical stretching vibration, respectively. These findings suggest that $\mathrm{P}(\mathrm{NIPAM}-\mathrm{co}-\mathrm{AA})$ has been successfully coated onto the surface of the UCNP. Furthermore, the exact amount of

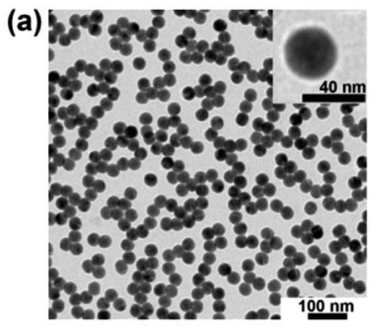

(c)

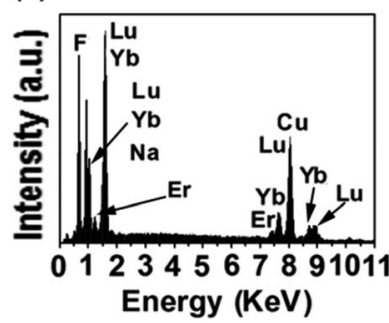

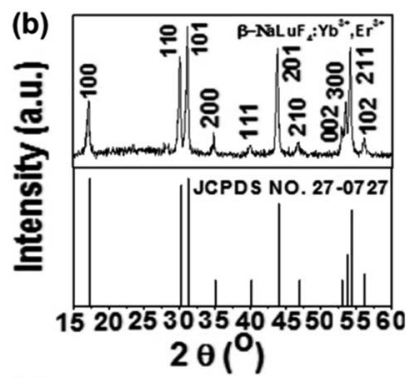

(d)

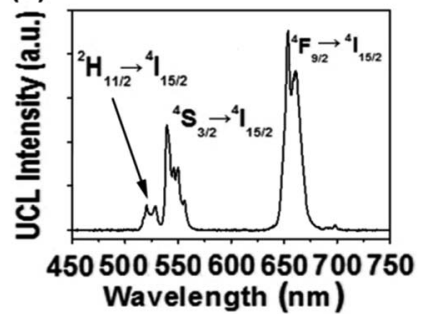

Fig. 1 (a) TEM image of UNCP@OA NaLuF $4: \mathrm{Yb}^{3+}, \mathrm{Er}^{3+}$; (b) X-ray power diffraction (XRD) spectra of the UNCP@OA hexagonal nanoparticles (JCPDS standard card no. 27-0727); (c) EDX spectra of UNCP@OA $\mathrm{NaLuF}_{4}: \mathrm{Yb}^{3+}, \mathrm{Er}^{3+}$; (d) upconversion emission spectra of OA-capped UNCPs under $980 \mathrm{~nm}$ laser excitation. polymer in each nanocomposites was determined by thermogravimetric analysis (TGA). TGA curves of the UCNP@OA and UCNP@P(NIPAM-co-AA) are illustrated in Fig. S2, $\uparrow$ the weight loss of OA and P(NIPAM-co-AA) on the surface of UCNP was $5 \%$ and $50 \%$, respectively. These results further infer that P(NIPAMco-AA) has successfully replaced OA on the surface of UCNPs by the ligand exchange process, and this finding agrees with the FTIR results.

The polymers PNIPAM selected for nanocomposite coating is a famous thermoresponsive polymer which has been widely used for intelligent multifunctional materials. The presence of PNIPAM chains anchored to the nanoparticle surface can undergo associate and aggeragation behavior to extend or shrink the polymer segment, thus induce the nanoparticle dispersing in aqueous solution below the LCST and precipitating above the LCST. The upconversion luminescence properties of UCNP@P(NIPAM-co-AA) were studied for preliminary experiment at the temperature of $27{ }^{\circ} \mathrm{C}$ and $31{ }^{\circ} \mathrm{C}$ under the excitation at $980 \mathrm{~nm}$. As a qualitative research, in the digital photograph (Fig. 2(c), inset), UCNP@P(NIPAM-co-AA) illuminates weak green light when excited at $27{ }^{\circ} \mathrm{C}$, however, the brightness of UCNP@P(NIPAM-co-AA) increase greatly at $31^{\circ} \mathrm{C}$.

In order to quantify the upconversion luminescence intensity of UCNP, UCL spectra were measured at different temperature from 27 to $34{ }^{\circ} \mathrm{C}$. At $27{ }^{\circ} \mathrm{C}$, there is mainly a strong peak centered at $670 \mathrm{~nm}$ and a weak peak centered at $545 \mathrm{~nm}$ in the visible region. The intensity at $670 \mathrm{~nm}$ is used as the standard and with the low value excited at $980 \mathrm{~nm}$ at same laser power below $31^{\circ} \mathrm{C}$. When temperature increase to $31^{\circ} \mathrm{C}$, the intensity increase sharply, a 80 -fold enhancement can be obtained with $670 \mathrm{~nm}$ intensity as a standard. The thermo-activatable temperature range of $1{ }^{\circ} \mathrm{C}$ indicates a remarkable thermoresponsive property which can be attributed to the sharp phase
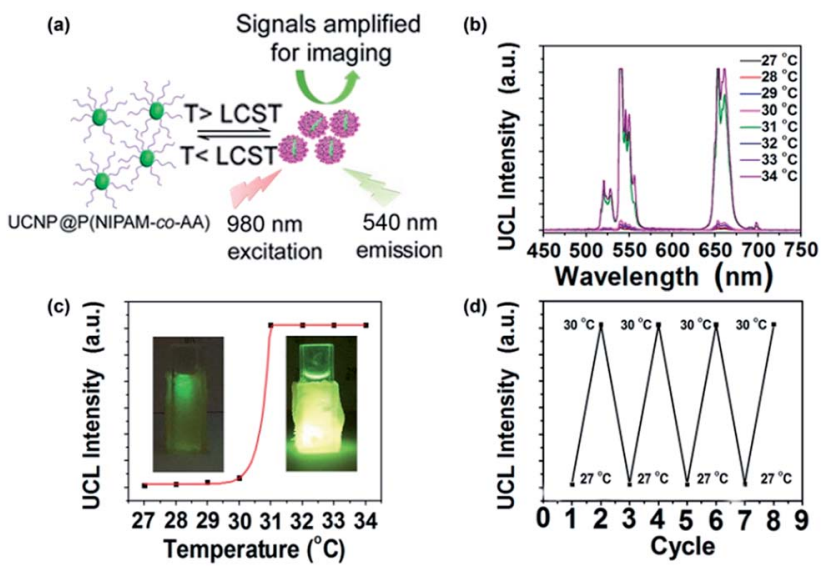

Fig. 2 (a)llustration of thermoresponsive upconversion emission of UCNP@P(NIPAM-CO-AA); (b)UCL spectrum of UCNP@P(NIPAM-CO-AA) dispersed in aqueous solution at different temperature under the excitation at $980 \mathrm{~nm}$; (c) UCL intensity as a function of temperature for UCNP@P(NIPAM-CO-AA) and the digital picture of UCNP@P(NIPAMco-AA) covered with $2 \mathrm{~mm}$ chicken; (d) several cycles of dynamic heating and cooling, showing the repeatability of the upconversion emission intensity with concentration $1 \mathrm{mg} \mathrm{mL}^{-1}$. 
transition of PNIPAM segment. A reversible thermosensitive properties is also observed in three cycle of heating and cooling process according to the reversible aggregation/disaggregation behavior of the PNIPAM segment above and below the LCST respectively (Fig. 2(d)), which indicates the nanoparticles can be used repeatedly and can be used for temperature-activatable UCL imaging agent.

\subsection{UCL imaging in vitro}

To investigate the temperature-activatable UCL imaging capability of the nanoparticles, we prepared a sample in a phantom where were filled with UCNP@P(NIPAM-co-AA) at $1 \mathrm{mg} \mathrm{mL} \mathrm{m}^{-1}$ and covered with a $2 \mathrm{~mm}$ thick chicken. The sample was excited with $980 \mathrm{~nm}$ NIR laser at different temperature. From $28{ }^{\circ} \mathrm{C}$ to $30{ }^{\circ} \mathrm{C}$, the UCL images showed an weak signal, however, a intense signal can be observed above $31{ }^{\circ} \mathrm{C}$ (Fig. 3(a)). We quantified the signal intensity in different regions of interest (ROI) over the background noise (Fig. 3(b)). At $28{ }^{\circ} \mathrm{C}$, the UCL signal-to-noise ratio (SNR) is only 1.6-fold for the UCNP@P(NIPAM-co-AA) nanocomposite.

Then we compared the contrast of UCL fluorescence images at $31{ }^{\circ} \mathrm{C}$, a 21 -fold SNR can be obtained for the same sample (Fig. 3(c)). With the temperature continuously increased, the SNR also increase slightly to 26 -fold at $34{ }^{\circ} \mathrm{C}$ which can be attributed to the sufficiently aggregation of the UCNP@P(NIPAM-co-AA) nanocomposite. The contrast ratio $\left(\mathrm{SNR}_{31}{ }^{\circ} \mathrm{C} / \mathrm{SNR}_{30}{ }^{\circ} \mathrm{C}\right)$ is 13.2 fold based on the UCL images, demonstrating that the UCL are highly responsive to the temperature changes in the environment.

\subsection{In vitro cytotoxicity}

The cytotoxicity of UCNP@P(NIPAM-co-AA) was carefully studied by the MTT protocol. As shown in Fig. 4, the viabilities of A549 cells were greater than $90 \%$ viable at the $24 \mathrm{~h}$ time point when the concentration of UCNP@P(NIPAM-co-AA) was less than $400 \mu \mathrm{g} \mathrm{mL}^{-1}$.

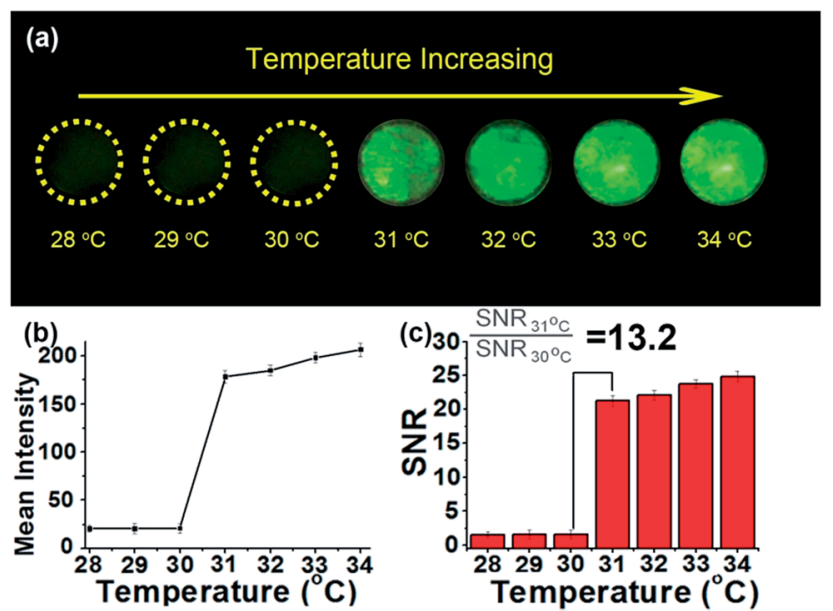

Fig. 3 (a) UCL images of UCNP@P(NIPAM-CO-AA) (1 $\mathrm{mg} \mathrm{mL}^{-1}$ ) phantom at different temperature; (b) intensity of UCL fluorescence for UCNP@ P(NIPAM-CO-AA) as a function of temperature; (c) comparison of SNR ratios from $28^{\circ} \mathrm{C}$ to $34^{\circ} \mathrm{C}$.

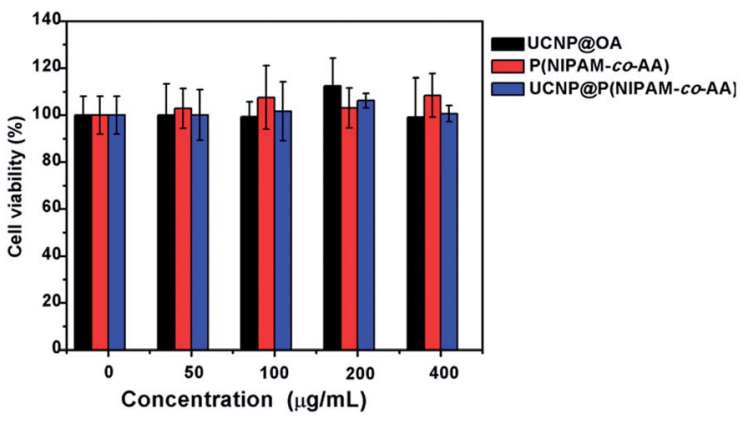

Fig. 4 Cytotoxicity test of UCNP@P(NIPAM-CO-AA) on the viability of A549 incubated with different concentrations of UCNP@P(NIPAM-CO-AA) as measured by MTT assay.

Therefore, UCNP@P(NIPAM-co-AA) can be considered to have a relatively low cytotoxicity at concentrations less than $400 \mu \mathrm{g} \mathrm{mL} \mathrm{L}^{-1}$.

\section{Conclusions}

In this work, we prepared intelligent temperature-activatable nanoparticles UCNP@P(NIPAM-co-AA) based on $\mathrm{NaLuF}_{4}: \mathrm{Yb}^{3+}$, $\mathrm{Er}^{3+}$ UCNPS coated with thermo-responsive copolymer $\mathrm{P}$ (NIPAM-co-AA). The polymer PNIPAM endows the UCNP with thermo-activatable properties to self-assemble reversibly, while retaining their upconversion emission properties in aqueous solution. Below the LCST, the UCNP@P(NIPAM-co-AA) shows weak up-conversion emission, and a 80-fold enhanced upconversion emission above $31{ }^{\circ} \mathrm{C}$. 13 -fold SNR can be obtained for in vitro imaging. The MTT results show low toxicity of the novel nanocomposite UCNP@P(NIPAM-co-AA) which could be extremely useful for future applications in bioimaging drug delivery and therapeutics.

\section{Conflicts of interest}

There are no conflicts to declare.

\section{Acknowledgements}

The project are grateful for the financial support from the National Natural Science Youth Foundation of China (No. 21204051), Beijing Education Committee Foundation program, and Technology Foundation for Selected Overseas Chinese Scholar, Ministry of Personnel of China.

\section{Notes and references}

1 Y. Dai, H. Xiao, J. Liu, Q. Yuan, P. Ma, D. Yang, C. Li, Z. Cheng, Z. Hou, P. Yang and J. Lin, J. Am. Chem. Soc., 2013, 135, 18920-18929.

2 G. Y. Chen, H. L. Qiu, P. N. Prasad and X. Y. Chen, Chem. Rev., 2014, 114, 5161-5214.

3 L. Z. Zhao, J. J. Peng, Q. Huang, C. Y. Li, M. Chen, Y. Sun, Q. N. Lin, L. Y. Zhu and F. Y. Li, Adv. Funct. Mater., 2014, 24, 363-371. 
4 F. Zhang, R. C. Haushalter, R. W. Haushalter, Y. F. Shi, Y. C. Zhang, K. L. Ding and D. Y. Zhao, Small, 2011, 7, 1972-1976.

5 D. Q. Chen, M. Xu, P. Huang, M. F. Ma, M. Y. Ding and L. Lei, J. Mater. Chem. C, 2017, 5, 5434-5443.

6 Y. M. Wu, Y. Cen, L. J. Huang, R. Q. Yu and X. Chu, Chem. Commun., 2014, 50, 4759-4762.

7 D. Q. Chen, M. Xu and P. Huang, Sens. Actuators, B, 2016, 231, 576-583.

8 D. Q. Chen and P. Huang, Dalton Trans., 2014, 43, 1129911304.

9 A. Xia, Y. Gao, J. Zhou, C. Y. Li, T. S. Yang, D. M. Wu, L. M. Wu and F. Y. Li, Biomaterials, 2011, 32, 7200-7208.

10 L. C. Ong, L. Y. Ang, S. Alonso and Y. Zhang, Biomaterials, 2014, 35, 2987-2998.

11 S. J. Zeng, H. B. Wang, W. Lu, Z. G. Yi, L. Rao, H. G. Liu and J. H. Hao, Biomaterials, 2014, 35, 2934-2941.

12 D. Q. Chen, L. Liu, P. Huang, M. y. Ding, J. S. Zhong and Z. G. Ji, J. Phys. Chem. Lett., 2015, 6, 2833-2840.

13 L. N. Sun, Z. W. Wei, H. G. Chen, J. L. Liu, J. J. Guo, M. Cao, T. Q. Wen and L. Y. Shi, Nanoscale, 2014, 6, 8878-8883.

14 L. Cheng, C. Wang and Z. Liu, Nanoscale, 2013, 5, 23-37.
15 A. Gnach and A. Bednarkiewicz, Nano Today, 2012, 7, 532563.

16 I. Craciun, G. Gunkel-Grabole, A. Belluati, C. G. Palivan and W. Meier, Nanomedicine, 2017, 12, 811-817.

17 M. Ghorbani and H. Hamishehkar, Int. J. Pharm., 2017, 520, 126-138.

18 B. Kang, A. Kukreja, D. Song, Y. M. Huh, S. Haam and S. Haam, J. Biol. Eng., 2017, 11, 13-25.

19 I. Dimitrov, B. Trzebicka, A. H. Muller, A. Dworak and C. B. Tsvetanov, Prog. Polym. Sci., 2007, 32, 1275-1343.

20 M. Yamato, Y. Akiyama, J. Kobayashi, J. Yang, A. Kikuchi and T. Okano, Prog. Polym. Sci., 2007, 32, 1123-1133.

21 H. G. Schild, Prog. Polym. Sci., 1992, 17, 163-249.

22 K. Suwa, K. Morishita, A. Kishida and M. Akashi, J. Polym. Sci., Part A: Polym. Chem., 1997, 35, 3087-3094.

23 Z. Q. Li and Y. Zhang, Nanotechnology, 2008, 19, 345606345611.

24 Z. Q. Li, Y. Zhang and S. Jiang, Adv. Mater., 2008, 20, 47654769.

25 F. Wang, Y. Han, C. S. Lim, Y. Lu, J. Wang, J. Xu, H. Chen, C. Zhang, M. Hong and X. Liu, Nature, 2010, 463, 1061-1065. 26 G. S. Yi and G. M. Chow, Adv. Funct. Mater., 2006, 16, 23242329. 